\title{
BMJ
}

\section{Radiation exposure and circulatory disease risk: Hiroshima and Nagasaki atomic bomb survivor data, 1950-2003}

\author{
Yukiko Shimizu, visiting research associate, ${ }^{1}$ Kazunori Kodama, chief scientist, ${ }^{2}$ Nobuo Nishi, assistant \\ department chief, ${ }^{1}$ Fumiyoshi Kasagi, assistant department chief, ${ }^{1}$ Akihiko Suyama, department chief, ${ }^{3}$ Midori \\ Soda, assistant department chief, ${ }^{3}$ Eric J Grant, associate senior scientist, ${ }^{1}$ Hiromi Sugiyama, research \\ scientist, ${ }^{1}$ Ritsu Sakata, research scientist, ${ }^{1}$ Hiroko Moriwaki, research assistant, ${ }^{1}$ Mikiko Hayashi, research \\ assistant, ${ }^{1}$ Manami Konda, research assistant, ${ }^{1}$ Roy E Shore, vice chairman and chief of research ${ }^{2}$
}

${ }^{1}$ Department of Epidemiology, Radiation Effects Research

Foundation, 5-2 Hijiyama Park, Minami-ku, Hiroshima 732-0815,

Japan

${ }^{2}$ Radiation Effects Research Foundation, Hiroshima

${ }^{3}$ Department of Epidemiology, Radiation Effects Research Foundation, Nagasaki 850-0013,

Japan

Correspondence to: $Y$ Shimizu shimizu@rerf.or.jp

Cite this as: $B M J$ 2010;340:b5349 doi:10.1136/bmj.b5349

\section{ABSTRACT}

Objective To investigate the degree to which ionising radiation confers risk of mortality from heart disease and stroke.

Design Prospective cohort study with more than 50 years of follow-up.

Setting Atomic bomb survivors in Hiroshima and Nagasaki, Japan.

Participants 86611 Life Span Study cohort members with individually estimated radiation doses from 0 to $>3 \mathrm{~Gy}$ ( $86 \%$ received $<0.2 \mathrm{~Gy}$ ).

Main outcome measures Mortality from stroke or heart disease as the underlying cause of death and doseresponse relations with atomic bomb radiation.

Results About 9600 participants died of stroke and 8400 died of heart disease between 1950 and 2003. For stroke, the estimated excess relative risk per gray was $9 \%(95 \%$ confidence interval $1 \%$ to $17 \%, \mathrm{P}=0.02$ ) on the basis of a linear dose-response model, but an indication of possible upward curvature suggested relatively little risk at low doses. For heart disease, the estimated excess relative risk per gray was $14 \%$ (6\% to $23 \%$, P<0.001); a linear model provided the best fit, suggesting excess risk even at lower doses. However, the dose-response effect over the restricted dose range of 0 to $0.5 \mathrm{~Gy}$ was not significant. Prospective data on smoking, alcohol intake, education, occupation, obesity, and diabetes had almost no impact on the radiation risk estimates for either stroke or heart disease, and misdiagnosis of cancers as circulatory diseases could not account for the associations seen.

Conclusion Doses above 0.5 Gy are associated with an elevated risk of both stroke and heart disease, but the degree of risk at lower doses is unclear. Stroke and heart disease together account for about one third as many radiation associated excess deaths as do cancers among atomic bomb survivors.

\section{INTRODUCTION}

The effects of radiation on incidence of or mortality from circulatory disease have large implications for public health, especially if effects occur at doses under $1 \mathrm{~Gy}$. Given that the frequency of multiple computed tomography scans of the head or chest and of interventional radiographic procedures is increasing rapidly, information on whether these may confer risk for subsequent stroke or heart disease is essential.

Several studies, including randomised controlled trials, have found that high doses of radiation to the heart from radiotherapy for Hodgkin's disease or breast cancer cause an excess of deaths from heart disease in later years, ${ }^{1-4}$ and other studies have suggested that radiotherapy for Hodgkin's disease, childhood leukaemia or brain tumours, and head and neck cancer increases the risk of stroke. ${ }^{5-8}$ Several authors have suggested that lower doses from occupational, medical, and environmental exposures may be associated with excess mortality from circulatory disease, ${ }^{9-14}$ although other studies have not found such low dose effects, ${ }^{15-19}$ and information on doses and potential confounding lifestyle factors is limited in many of the studies of low doses. We examined the dose-response information on the risk of heart disease and stroke in the large Life Span Study cohort of atomic bomb survivors in Hiroshima and Nagasaki who have been followed up for 53 years, from 1950 to 2003.

\section{METHODS}

\section{Study population}

The Life Span Study cohort, defined on the basis of the Japanese national census in 1950 and special surveys between 1950 and 1953, consists of 86611 atomic bomb survivors with estimated radiation doses. It includes a large proportion of the survivors who were within $2.5 \mathrm{~km}$ of the hypocentres at the time of the bombings and still resided in Hiroshima or Nagasaki in 1950, plus a random age and sex matched sample of people 2.5 to $10 \mathrm{~km}$ from the hypocentre who sustained small to negligible radiation doses. ${ }^{20}$ This study population was of all ages and both sexes at the time of the bombings.

Individual doses have been carefully estimated using the recent improved DS02 dosimetry system, 
primarily on the basis of people's location and shielding at the time of the atomic bomb. ${ }^{2122}$ We estimated risks by using weighted colon doses in gray $(\mathrm{Gy})$ for all analyses. We used weighted doses, the sum of the $\gamma$ dose plus 10 times the smaller neutron dose, to allow for the greater biological effectiveness of neutrons.

The follow-up of vital status took place from 1 October 1950 to the end of 2003 and was based on the nationwide obligatory family registration system (koseki) that documents mortality and is virtually $100 \%$ complete. Causes of death came from the official vital statistics death schedules based on the death certificates. Underlying and contributing causes of death were classified according to the ICD-7 (international classification of diseases, 7th revision) (for deaths in 1950-68), ICD-8 (in 1969-78), ICD-9 (in 1979-97), and ICD-10 (in 1998-2003). However, for the purposes of these analyses we converted them to ICD-9 codes 390-459 for all circulatory disease, $430-438$ for stroke, and 393-429 (excluding 401, 403, and 405) for heart disease. We used only underlying causes of death in the primary analyses but examined underlying plus contributing causes in a subsidiary analysis.

\section{Collection of covariate data and data from autopsy and tumour registry}

A mail survey was sent to a defined sub-cohort of 51965 Life Span Study cohort members in 1978. Information was obtained from 36468 (response rate of $70 \%$ ) on sociodemographic (education, type of occupation), lifestyle (smoking, alcohol intake), and health variables (obesity, diabetes mellitus), which enabled the evaluation of possible confounding by these variables. Between 1950 and 1985 autopsy data were also available on more than 1900 deaths that had an underlying cause of circulatory disease on the death certificate, which permitted evaluation of diagnostic accuracy. To identify pre-existing cases of cancer, we used the Hiroshima and Nagasaki tumour registries (available since 1958) and tissue registries (since 1974).

\section{Statistical analysis}

We based the analyses on a detailed summary table of the number of deaths and person years stratified by dose, city, sex, and five year intervals of age at exposure, attained age, and follow-up period. We divided participants into categories according to the weighted colon dose (in $\mathrm{Gy}=\gamma$ dose plus 10 times neutron dose): 0-, 0.005-, 0.02-, 0.04-, 0.06-, 0.08-, 0.1-, 0.125-, 0.15-, 0.175-, 0.2-, 0.25-, 0.3-, 0.5-, 0.75-, 1-, 1.25-, 1.5-, 1.75-, $2-, 2.5-$, and $\geq 3$. As described elsewhere, we truncated the colon doses to correspond to the 4 Gy shielded kerma level, ${ }^{20}$ but this affected only 317 participants.

We used Poisson regression methods for grouped survival data to describe the dependence of risk on radiation dose and to evaluate the variation of the dose-response effects with respect to city, sex, age at exposure, time since exposure, and attained age, ${ }^{23}$ essentially identical to the methods used previously to examine mortality from cancer in this cohort. ${ }^{20} \mathrm{We}$ used Epicure software for parameter estimation and tests, ${ }^{24}$ and we based significance tests and 95\% confidence intervals on likelihood profiles.

The primary models used here are excess relative risk (ERR) models of the form $\lambda_{0}(\mathrm{c}, \mathrm{s}, \mathrm{a}, \mathrm{b})[1+\mathrm{ERR}(\mathrm{d}$, e,s,a)], where $\lambda_{0}()$ is the baseline, or background death rate (that is, the rate for people with zero dose), which depended on city $(\mathrm{c}), \operatorname{sex}(\mathrm{s})$, attained age (a), and birth year (b). The function ERR (d,e,s,a) describes the relative change in rates associated with dose (d), allowing for the effects of sex, age at exposure (e), and attained age. We examined effect modifiers by using models corresponding to those in Preston et al. ${ }^{2025} \mathrm{We}$ examined both dose and dose squared terms to evaluate the degree of linearity or curvature in the dose-response forms. We also tested a linear threshold model. We used differences in maximum likelihood to compare nested models or the Akaike information criterion for non-nested models. ${ }^{26}$ We evaluated a linear threshold model repeatedly for a wide range of possible values of a threshold dose $\left(\mathrm{d}_{0}\right)$, modelling the risk function ERR on doses $d$ as $\beta\left(d-d_{0}\right)$ for $d>d_{0}$ or $d=0$ for $d \leq d_{0}$. We empirically determined the values yielding the maximum likelihood and 95\% confidence bounds.

We examined the impact of the possible confounding factors of smoking (never, past, present $<20 /$ day, present $\geq 20 /$ day), alcohol intake (regular, seldom/ never), education (primary or less, secondary, college/university), occupation for household (professional/technical, clerical/sales, farmer/craftsman, transportation/service), obesity (body mass index $<20,20-24, \geq 25$ ), and diabetes (yes, no) on the estimates of radiation risk, including codes for missing information, for the Life Span Study participants included in the 1978 mail survey. We included Coxtype regression models fitted to the individual data, where radiation dose was modelled as a linear excess relative risk, and indicator variables for the potential confounders jointly in the models as conventional exponential relative risk terms by using the Peanuts program in Epicure. ${ }^{24}$

\section{RESULTS}

During follow-up, 19054 deaths from circulatory disease occurred among the 86611 Life Span Study members with DS02 dose available. Table 1 shows the numbers of participants and deaths from circulatory disease by age, sex, and radiation dose. The cohort covers a wide range of doses but is weighted towards low doses, which indicates that it has considerable capability to examine risks at low doses and to examine the shape of the dose-response curve. The deaths included 9622 from stroke, 8463 from heart disease, and 969 from other circulatory diseases. The excess relative risk per Gy for all circulatory disease based on the linear model over the full dose range was 11\% (95\% confidence interval $5 \%$ to $17 \%, \mathrm{P}<0.001)$. This represents about 210 excess cases of death from circulatory disease associated with the exposure to radiation. 
Table 1| Number of participants and deaths from circulatory disease

\begin{tabular}{|c|c|c|c|c|c|}
\hline \multirow[b]{2}{*}{ Characteristics } & \multirow[b]{2}{*}{$\begin{array}{c}\text { No of people } \\
(n=86611)\end{array}$} & \multicolumn{4}{|c|}{ No of deaths } \\
\hline & & $\begin{array}{l}\text { Circulatory disease } \\
\qquad(n=19054)\end{array}$ & $\begin{array}{c}\text { Stroke } \\
(n=9622)\end{array}$ & $\begin{array}{l}\text { Heart disease } \\
(n=8 \text { 463) }\end{array}$ & $\begin{array}{l}\text { Other circulatory } \\
\text { disease }(n=969)\end{array}$ \\
\hline \multicolumn{6}{|l|}{ Sex: } \\
\hline Male & 35687 & 7607 & 3958 & 3261 & 388 \\
\hline Female & 50924 & 11447 & 5664 & 5202 & 581 \\
\hline \multicolumn{6}{|c|}{ Age at atomic bomb exposure (years): } \\
\hline $0-9$ & 17833 & 428 & 176 & 238 & 14 \\
\hline $10-19$ & 17563 & 951 & 404 & 508 & 39 \\
\hline $20-29$ & 10891 & 1551 & 652 & 831 & 68 \\
\hline $30-49$ & 25774 & 9712 & 4735 & 4575 & 402 \\
\hline$\geq 50$ & 14550 & 6412 & 3655 & 2311 & 446 \\
\hline \multicolumn{6}{|c|}{ Weighted colon radiation dose (mGy): } \\
\hline$<5$ & 38509 & 8440 & 4247 & 3723 & 470 \\
\hline $5-$ & 23427 & 5089 & 2637 & 2205 & 247 \\
\hline $50-$ & 12508 & 2838 & 1405 & 1305 & 128 \\
\hline $200-$ & 6356 & 1485 & 735 & 680 & 70 \\
\hline $500-$ & 3424 & 745 & 363 & 342 & 40 \\
\hline $1000-$ & 1763 & 341 & 176 & 158 & 7 \\
\hline$\geq 2000$ & 624 & 116 & 59 & 50 & 7 \\
\hline
\end{tabular}

Stroke

The excess relative risk per Gy for stroke based on the linear model over the full dose range was $9 \%(1 \%$ to $17 \%, \mathrm{P}=0.02$ ) (table 2). Figure 1 shows estimates of the shape of the dose-response curve for all stroke, including the fitted linear and linear-quadratic models. The test for non-linearity based on a comparison of linear and linear-quadratic dose-response models was not statistically significant $(\mathrm{P}=0.17)$, but the pure quadratic model, which suggests relatively little risk at lower doses, nominally provided a slightly better fit (difference in Akaike information criterion statistics of 1.87) than did the linear model. This was confirmed by analyses of lower dose ranges which showed excess relative risk per Gy of 3\% (-10\% to $16 \%)$ for $0-1$ Gy and $-7 \%(-28 \%$ to $16 \%)$ for $0-0.5$ Gy. Figure 1 also shows no apparent risk for the lower part of the dose range; a non-negligible threshold may exist below which no excess occurs. The best estimate of a threshold dose was $0.5 \mathrm{~Gy}$ with an upper $95 \%$ confidence limit of about 2 Gy. However, the lower 95\% confidence limit was not greater than 0 , so no threshold dose may exist.

An analysis of effect modification of the risk of stroke by sex, attained age, and age at exposure showed a statistically significant difference for attained age $(\mathrm{P}=0.04)$ : the radiation excess relative risk per $\mathrm{Gy}$ for stroke was higher before age 60 than after, especially among men (web table A). We also found a non-significant indication $(\mathrm{P}=0.23)$ that the risk of stroke associated with radiation may be highest after exposure at young ages: the excess relative risk per Gy were 36\%, $9 \%, 15 \%$, and $5 \%$ for ages $<10,10-19,20-39$, and $\geq 40$. An evaluation of subtypes of stroke was not very meaningful because, before the 1990s, differential diagnosis was often not done, resulting in many cases being classified as stroke, not otherwise specified.

\section{Heart disease}

The excess relative risk per Gy for all heart disease based on the linear model in the full dose range was $14 \%(6 \%$ to $23 \%, \mathrm{p}<0.001)$ (table 2). Figure 2 shows the results for the linear and linear-quadratic models. The test for non-linearity based on a comparison of linear and linear-quadratic dose-response models was not statistically significant $(\mathrm{P}>0.5)$. A pure linear model fitted the data nominally better than did a pure dosesquared model (difference in Akaike information criterion statistics of 2.47). The excess relative risks per Gy for heart disease over restricted dose ranges were similar to that for the full dose range. Specifically, the excess relative risk per Gy based on the linear model for the dose ranges under 2, 1, and 0.5 Gy were $14 \%$ ( $4 \%$ to $25 \%$ ), $18 \%$ (3\% to $33 \%$ ), and $20 \%(-5 \%$ to $45 \%$ ). In figure 2 , the slope over the lower part of the dose range was almost identical to the one for the entire dose

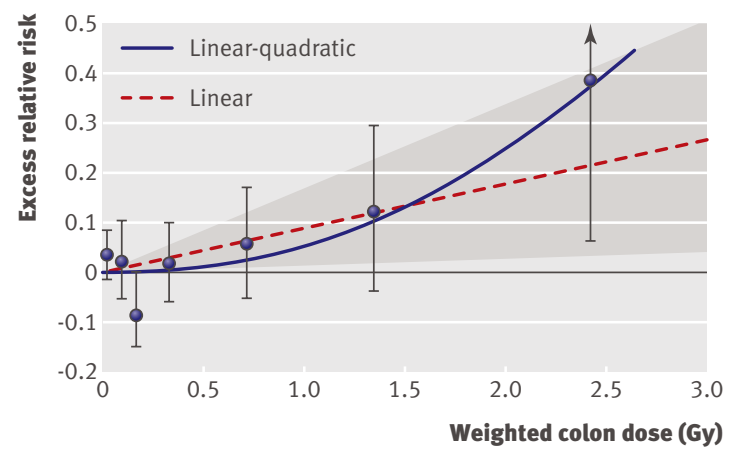

Fig 1| Radiation dose-response relation (excess relative risk per Gy) for death from stroke, showing linear and linearquadratic functions. Shaded area is $95 \%$ confidence region for fitted linear line. Vertical lines are $95 \%$ confidence intervals for specific dose category risks. Point estimates of risk for each dose category are indicated by circles 
Table 2 | Summary excess relative risks (ERR)* per Gy and excess additive risks per $10^{4}$ person year Gy† (EAR/10 4 PY-Gy) for types of circulatory disease mortality

\begin{tabular}{|c|c|c|c|c|c|c|}
\hline \multirow{2}{*}{$\begin{array}{l}\text { Circulatory } \\
\text { disease }\end{array}$} & \multicolumn{4}{|c|}{ Indicated as underlying cause of death } & \multicolumn{2}{|c|}{ Underlying or contributing cause of death } \\
\hline & Deaths & $P$ value & $\%$ ERR/Gy $(95 \% \mathrm{Cl})$ & EAR/104 PY-Gy $(95 \% \mathrm{Cl}) \dagger$ & Deaths & $\%$ ERR/Gy $(95 \% \mathrm{Cl})$ \\
\hline Total & 19054 & $<0.001$ & 11 (5 to 17$)$ & $5.5(2.7$ to 8.4$)$ & 25113 & 15 (10 to 20$)$ \\
\hline Stroke & 9622 & 0.02 & $9(1$ to 17$)$ & $2.3(0.4$ to 4.4$)$ & 12139 & 12 (5 to 19$)$ \\
\hline Heart disease & 8463 & $<0.001$ & 14 (6 to 23$)$ & $3.2(1.3$ to 5.2$)$ & 14018 & 18 (11 to 25$)$ \\
\hline Other & 969 & $>0.5$ & $2(-18$ to 29$)$ & $0.1(-0.4$ to 0.7$)$ & 5846 & $58(45$ to 72$)$ \\
\hline
\end{tabular}

*Estimates based on linear model, adjusted for city, sex, age at exposure, and attained age.

†Average EARs calculated directly from fitted ERR models.

range. The best estimate of a threshold dose was $0 \mathrm{~Gy}$, with an upper $95 \%$ confidence limit of about $0.5 \mathrm{~Gy}$. We found no significant modification of effect by sex, age at exposure, or age at risk (web table A).

Analyses of different subtypes of heart disease revealed some diversity in dose-response effects (web table B) but involve a variety of uncertainties, the articulation of which is beyond the scope of this report. The risk of ischaemic heart disease increased only in the higher dose categories, and the linear increase was not significant. We found stronger associations between radiation and other heart diseases, such as hypertensive heart disease and heart failure. However, unlike the relatively high accuracy in diagnosing the general category of heart disease, substantial misclassification of subtypes of heart disease occurs (see below), which limits the meaning that can be attached to the analyses of subtypes.

\section{Confounding factors and misdiagnosis}

We examined the impact of the possible confounding factors on the radiation risk estimates among the 51965 Life Span Study participants included in the 1978 mail survey. Table 3 shows the excess relative risks unadjusted and adjusted for six potential confounding factors. Note that the excess relative risks in table 3 differ slightly from those in table 2 , because the estimates in table 3 are based on only the subcohort of the Life Span Study, whereas those in table 2 are based on the full cohort (86611 participants). Although smoking, alcohol intake, education, type of occupation, obesity (body mass index), and diabetes were risk factors for heart disease and stroke in their own right (for example, the relative risks for heart disease were 1.4 for smoking, 1.6 for diabetes, 1.1 for body mass index 25 or over, and 0.75 for university education), they showed virtually no confounding with dose of radiation. That is, adjustment for the six variables simultaneously produced inconsequential changes in the excess relative risk per Gy: only $0.1 \%$ for heart disease and $-0.9 \%$ for stroke (table 3 ). Analyses limited to respondents similarly showed little impact of the confounder variables (data not shown). These results suggest that in the Life Span Study, the associations of dose of radiation with mortality from stroke and heart disease is unlikely to be an artefact of confounding by major lifestyle, sociodemographic, or disease risk factors.
We also examined the diagnostic accuracy of death certificates by comparing them with autopsy reports among the 1963 cases with death certificate designated circulatory disease for whom autopsies were available from our autopsy programme between 1950 and $1985 .{ }^{27}$ For the broad categories of stroke and heart disease, the accuracy of the diagnoses on the death certificates was fairly good. For death certificates with stroke as the underlying cause of death, $86 \%$ of autopsies listed stroke as a cause; $92 \%$ of death certificates with heart disease as the underlying cause had heart disease listed as a cause on the autopsy report. Moreover, the accuracy of diagnoses on death certificates has probably improved since 1985 . However, the corresponding accuracy was rather poor for the differential diagnosis of specific subcategories of stroke or heart disease (for example, 65\% for cerebral infarction, $39 \%$ for cerebral haemorrhage, $69 \%$ for ischaemic heart disease, $22 \%$ for hypertensive heart disease, and $64 \%$ for rheumatic heart disease); web table B shows risk estimates for separate subcategories.

Deaths from circulatory disease based on death certificates may include misdiagnosed deaths from cancer or cases arising from cardiotoxicity due to chemotherapy or radiotherapy for cancer. To remove the effects of misdiagnosis of cases of cancer, we estimated risks after excluding people who had previous diagnoses of cancer, on the basis of our tumour registry data. These excess relative risks were reduced by about 30\% compared with estimates based on the full cohort, but still

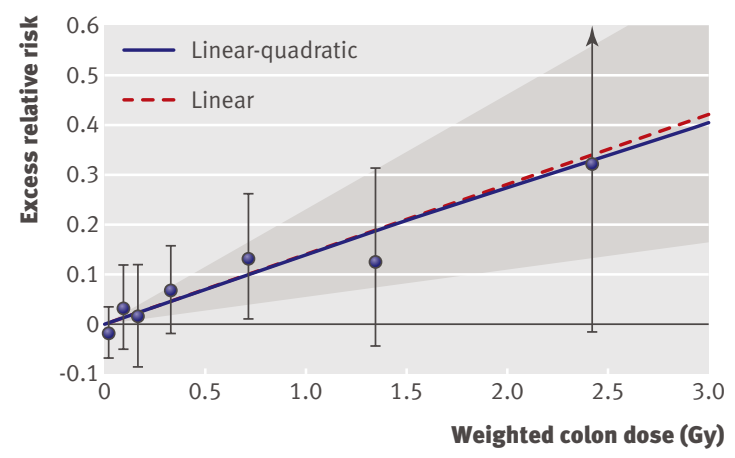

Fig 2 | Radiation dose-response relation (excess relative risk) for death from heart disease, showing linear and linearquadratic functions. Shaded area is $95 \%$ confidence region for fitted linear line. Vertical lines are $95 \%$ confidence intervals for specific dose category risks. Point estimates of risk for each dose category are indicated by circles 
Table $3 \mid$ Effects of potential confounding factors on radiation risk estimates for types of circulatory disease mortality

\begin{tabular}{lccc} 
Circulatory disease & No of deaths & $\begin{array}{c}\text { \% ERR/Gy unadjusted } \\
\text { for confounders* }\end{array}$ & $\begin{array}{c}\text { \% ERR/Gy adjusted } \\
\text { for all confounders* }\end{array}$ \\
Total & 7907 & 10.0 & 9.6 \\
\hline Stroke & 3366 & 8.1 & 7.2 \\
\hline Heart disease & 4204 & 12.2 & 12.3 \\
\hline Other & 337 & 2.4 & 0.9 \\
\hline
\end{tabular}

$E R R=e x c e s s$ relative risks.

*All analyses adjusted for city, sex, age at exposure, and attained age.

†Additionally adjusted for smoking, alcohol intake, education, type of household occupation, obesity (body mass index), and diabetes mellitus (on basis of about 52000 participants).

showed a tendency to significant dose-response effects. Excluding previous cases of cancer changed the excess relative risk per Gy from $10.8 \%$ to $7.3 \%$ (dose-response $\mathrm{P}=0.008)$ for all circulatory disease, from $8.8 \%$ to $6.2 \%$ $(\mathrm{P}=0.11)$ for stroke, and from $14.0 \%$ to $9.5 \%(\mathrm{P}=0.03)$ for heart disease.

Although we used the designated underlying cause of death in the mortality analyses, selecting a single cause of death is difficult when several correlated diseases or conditions contributed to death. Therefore, we examined the risks on the basis of both underlying and contributing causes of death (table 2). The radiation dose-response effects were nominally higher than those based on underlying cause of death alone $(12 \%$ $v 9 \%$ excess relative risk per Gy for stroke, and 18\% v $14 \%$ for heart disease), which lends additional support to the hypothesis of radiation risk.

\section{DISCUSSION}

This study found dose-response evidence for risk of heart disease and stroke among atomic bomb survivors over the radiation dose range 0-4 Gy (mostly 0-2 Gy) based on well characterised individual doses and essentially complete ascertainment of mortality over the period of five to 58 years after exposure to radiation. This report updates earlier brief reports of a dose related excess of circulatory disease among atomic bomb survivors. ${ }^{202829}$ These results, based on about $25 \%$ more deaths than the previous paper, are substantially stronger, and we now provide more elaboration of the associations.

As shown in table 1, at the youngest ages of exposure (more recent birth cohorts) the deaths from heart disease outnumber those from stroke, whereas the opposite is true of the earlier birth cohorts; this reflects the general secular trends in the Japanese population. The table also shows that the cohort covers a wide range of doses but is weighted toward low doses, indicating that it has substantial capability to examine risks at low doses and to examine the shape of the dose-response curve. Because several plausible disease mechanisms centre around systemic effects after whole body irradiation, we used colon doses as an approximation to whole body doses for all analyses, although analyses using brain dose for stroke and lung dose for heart disease produced very similar results (data not shown).
Summary of features and coherence of radiation risk data Although the data were statistically consistent with linearity over the full dose range in this study, considerable uncertainty exists about the shape of the doseresponse curve in the low dose range. The extent of curvature seemed to be larger for stroke than for heart disease; a pure dose-squared model fitted the stroke data slightly better than did a pure linear model, whereas the linear model provided a better fit for heart disease. However, the dose-response effect was not statistically significant for either end point when we limited the calculation to the dose range 0$0.5 \mathrm{~Gy}$, implying that evidence is limited on the risk below about $0.5 \mathrm{~Gy}$. For stroke, the estimated threshold dose was $0.5 \mathrm{~Gy}$, with an upper $95 \%$ confidence limit of about 2 Gy. For heart disease, the estimated threshold dose was $0 \mathrm{~Gy}$, with an upper $95 \%$ confidence limit of about $0.5 \mathrm{~Gy}$.

Additional analyses supported the association of radiation with stroke and heart disease. Adjustment of the data for other potential risk factors for circulatory disease - obesity, diabetes, smoking, alcohol consumption, education, and occupation - had almost no impact on the associations with radiation, whereas an analysis for possible misdiagnosis of cancer as circulatory disease showed a small diminution of radiation risk. Because the underlying cause of death is often uncertain, we also did analyses of stroke and heart disease indicated as either an underlying or contributing cause; these showed nominally stronger associations with radiation than did the analyses of underlying cause alone.

The findings of the epidemiological study of circulatory disease among atomic bomb survivors are confirmed and extended by our Adult Health Study, which consists of biennial clinical and laboratory examinations since 1958 of about 15\% of the Life Span Study cohort members. The Adult Health Study has found dose related increases in the incidence of stroke and myocardial infarction and in the incidence or prevalence of hypertension, elevated serum cholesterol concentrations, and aortic arch calcification. ${ }^{30-35}$ Late radiation effects have also been found for potential biomarker precursors of circulatory disease, including biomarkers for inflammation, ${ }^{36-38}$ deficient immunological responses, ${ }^{39}$ and alterations in immune cell repertoire. ${ }^{4041}$ The findings present a reasonably coherent picture of preclinical and clinical risk of circulatory disease associated with exposure to radiation. However, this needs to be complemented by a risk assessment of low doses based on mechanistic and animal models.

\section{Strengths and limitations of study}

This study has several strengths, including a large population not pre-selected for existing disease or occupational fitness, a wide but relatively low dose range $(0->3 \mathrm{~Gy})$ and well characterised doses, a 53 year follow-up with virtually complete mortality ascertainment, and corroborative evidence from 
more detailed clinical and biomarker studies of risk of circulatory disease on a random subsample of the cohort. ${ }^{30-41}$ In addition, we believe medical surveillance bias to be minimal, as all of the cohort is eligible for free, special medical care, and many people have little idea of the doses they received, so that the level of radiation related medical concern is not highly correlated with the actual dose received. In addition, the analyses of radiation dose with stroke and heart disease mortality showed that the association is reasonably robust with respect to confounding by lifestyle, sociodemographic, or other health factors or misdiagnosis.

The study also has several limitations and uncertainties. Ascertainment of circulatory disease from death certificates is of limited diagnostic accuracy and represents only a fraction of cases of incident disease. Analyses for confounders, although very important, are incomplete, lacking information on, for example, blood lipids, physical activity, and nutrition. Some selection effects due to dose related early mortality from the bombs may have occurred, although the impact of these is likely to be small. ${ }^{42}$ Other limitations include unclear dose-response effects below about 0.5 Gy, inadequate information about possible biological mechanisms, and uncertainty about the generalisability of these results to Western populations because of differences in genetic factors, dietary and lifestyle risk factors, and baseline levels of risk for stroke and heart disease. $^{43}$

\section{Comparison with other studies}

Although epidemiological and experimental data are limited, several studies suggest the possibility of effects of radiation on circulatory disease. Among medically exposed cohorts, excess heart disease mortality has been shown among patients who received radiotherapy for Hodgkin's lymphoma or breast cancer. ${ }^{1-44}$ At somewhat lower doses, an increase in coronary heart disease was seen among patients who received radiotherapy for peptic ulcer. ${ }^{9}$ An association was also seen among patients with scoliosis who received multiple fluoroscopic examinations, ${ }^{45}$ but not among patients with tuberculosis who received multiple fluoroscopic examinations of the chest,$^{16}$ nor among patients who received radiographic treatment for benign gynaecological disease. ${ }^{4647}$

Studies of cohorts with occupational or environmental exposure to radiation have not provided clear evidence for or against a radiation associated increase in mortality from circulatory disease. In a long term follow-up of early US radiologists, circulatory disease mortality was higher than for a comparison group of other medical specialists, ${ }^{48}$ but a similar increase was not seen among early UK radiologists. ${ }^{17}$ Increased mortality from circulatory disease was found among US radiological technologists who worked before 1950, when radiation exposures tended to be higher, but individual people's doses were not documented. ${ }^{10}$ Significant associations with radiation for both stroke and heart disease were reported for emergency workers at Chernobyl, ${ }^{12}$ although the study may have limitations related to sample selection and to variations in circulatory disease risk factors and medical surveillance. Among workers for British Nuclear Fuels, a significant dose-response association was found for ischaemic heart disease but not cerebrovascular disease. ${ }^{13}$ No association was found between radiation dose and circulatory diseases among German uranium miners. ${ }^{19}$ Preliminary results for the Mayak nuclear workers show a statistically significant dose-response association for ischaemic heart disease and stroke. ${ }^{49}$ A new update of the UK national registry for radiation workers shows a marginally positive association of dose of radiation with heart disease, but further analyses suggest that the finding might be due to variations in smoking habits. ${ }^{14}$ An analysis of combined cohorts of 275000 nuclear workers from 15 countries who were exposed to low, well documented doses of external radiation did not show a significant association between dose of radiation and either stroke or heart disease. $^{15}$

With the exception of the study of US radiological technologists, the studies were not able to adjust for potential lifestyle factors or other confounding factors, and some of the studies had no or only crude estimates of individual doses. Most of the studies of low doses had limited statistical power and some potential for bias; consequently, the potential for both false positive and false negative results may be high. The United Nations Scientific Committee on the Effects of Atomic Bomb Radiation (UNSCEAR) concluded that little evidence, other than the atomic bomb studies, exists to support an association between circulatory disease and radiation in the dose range less than $1-2 \mathrm{~Gy} .{ }^{50} \mathrm{~A}$ recent review article reached a similar conclusion, ${ }^{51}$ although additional suggestively positive data have appeared since these reviews were written. ${ }^{13} 149$

\section{Mechanisms of circulatory disease}

Knowledge of the mechanisms by which radiation doses of 2 Gy or less may cause circulatory disease is limited. Evidence suggests that pro-inflammatory responses to radiation, cellular loss or functional changes in the endothelium, or microvascular damage may be early events in the cascade of pathogenic changes that lead to radiation related heart disease..$^{52-55}$ These may augment other risk factors, such as hypertension, high serum cholesterol, smoking, diabetes, and infection, to promote heart disease.$^{56}$ Associations between dose of radiation and long term levels of inflammatory markers have been documented among atomic bomb survivors, ${ }^{36-38}$ possibly because of damage to the immune system. ${ }^{39}$ Radiation associated microvascular damage to the renal parenchyma and vascular endothelial cells may promote hypertension and ischaemia. ${ }^{50.57}$

\section{Conclusions and implications}

The effect of radiation on risk of circulatory disease is potentially a very important public health concern. 


\section{WHAT IS ALREADY KNOWN ON THIS TOPIC}

High doses of radiation to the heart or head and neck from radiotherapy cause a subsequent excess of deaths from heart disease or stroke

Whether radiation exposures at dose levels under $1 \mathrm{~Gy}$ also increase the risk of heart disease or stroke is not known

\section{WHAT THIS STUDY ADDS}

Radiation may increase the rates of stroke and heart disease at moderate dose levels (mainly 0.5-2 Gy), although the results below 0.5 Gy were not statistically significant

The association was reasonably robust with respect to confounding by lifestyle, sociodemographic, or other health factors or misdiagnosis

Given the widespread use of multiple computed tomography scans, ${ }^{559}$ and other relatively high dose diagnostic medical procedures, as well as radiotherapy that exposes the heart, the implications are substantial insofar as effects occur at doses under $1 \mathrm{~Gy}$. The potential magnitude of the risk is shown by the fact that in the Life Span Study cohort, who received whole body irradiation, the radiation related excess of deaths from circulatory diseases (about 210) is about a third as large as the total excess number of deaths from cancer (about $625)$.

This study provides the strongest evidence available to date that radiation may increase the rates of stroke and heart disease at moderate dose levels (mainly 0.5-2 $\mathrm{Gy}$ ), but robust confirmatory evidence from other studies is needed. Although our results below 0.5 Gy are not statistically significant, the additional cases occurring with further follow-up time should provide more precise estimates of the risk at low doses.

We thank the members of Radiation Effects Research Foundation Master File section for their diligent efforts to provide accurate data on mortality in the Life Span Study and Hiroshima and Nagasaki tumour and tissue registries for their approval for the use of the data. We also thank Yoshisuke Nonaka for statistical advice.

Contributors: YS, RES, KK, NN, FK, EJG, HS, RS, AS, and MS contributed to the study concept and design and to writing the paper. NN, HS, and MS advised on the tumour registry data. $\mathrm{HM}, \mathrm{MH}$, and MK contributed to data preparation for statistical analyses. All authors participated in interpreting the results. YS is the guarantor.

Funding: The Radiation Effects Research Foundation (RERF), Hiroshima and Nagasaki, Japan is a private, non-profit foundation funded by the Japanese Ministry of Health, Labour and Welfare (MHLW) and the US Department of Energy (DOE), the latter in part through the National Academy of Sciences. This publication was supported by RERF research protocol 1-75. The views expressed in this paper are those of the authors alone.

Competing interests: None declared.

Ethical approval: The research was conducted under the formal approval of RERF's Human Investigation Committee.

Data sharing: Detailed tabulation of data used for the analysis and the statistical code are available from the corresponding author.

1 Swerdlow AJ, Higgins CD, Smith P, Cunningham D, Hancock BW, Horwich A, et al. Myocardial infarction mortality risk after treatment for Hodgkin disease: a collaborative British cohort study. J Natl Cancer Inst 2007;99:206-14.

2 Darby SC, McGale P, Taylor CW, Peto R. Long-term mortality from heart disease and lung cancer after radiotherapy for early breast cancer: prospective cohort study of about 300,000 women in US SEER cancer registries. Lancet Oncol 2005;6:557-65.

3 Darby S, McGale P, Peto R, Granath F, Hall P, Ekbom A. Mortality from cardiovascular disease more than 10 years after radiotherapy for breast cancer: nationwide cohort study of 90,000 Swedish women. BMJ 2003;326:256-7.

4 Early Breast Cancer Trialists Collaborative Group (EBCTCG). Effects of radiotherapy and of differences in the extent of surgery for early breast cancer on local recurrence and 15-year survival: an overview of the randomized trials. Lancet 2005;366:2087-106.

5 Bowers DC, McNeil DE, Liu Y, Yasui Y, Stovall M, Gurney JG, et al. Stroke as a late treatment effect of Hodgkin's disease: a report from the Childhood Cancer Survivor Study. I Clin Oncol 2005;23:6508-15.

6 De Bruin ML, Dorresteijn LD, van't Veer MB, Krol AD, van der Pal HJ, Kappelle AC, et al. Increased risk of stroke and transient ischemic attack in 5-year survivors of Hodgkin lymphoma. J Natl Cancer Inst 2009;101:928-37.

7 Bowers DC, Liu Y, Leisenring W, McNeil E, Stovall M, Gurney JG, et al. Late-occurring stroke among long-term survivors of childhood leukemia and brain tumors: a report from the Childhood Cancer Survivor Study. I Clin Oncol 2006;24:5277-82.

8 Smith GL, Smith BD, Buchholz TA, Giordano SH, Garden AS, Woodward WA, et al. Cerebrovascular disease risk in older head and neck cancer patients after radiotherapy. J Clin Oncol 2008;26:5119-25.

9 Carr ZA, Land CE, Kleinerman RA, Weinstock RW, Stovall M, Griem ML, et al. Coronary heart disease after radiotherapy for peptic ulcer disease. Int J Radiat Oncol Biol Phys 2005;61:842-50.

10 Hauptmann M, Mohan AK, Doody MM, Linet MS, Mabuchi K. Mortality from diseases of the circulatory system in radiologic technologists in the United States. Am J Epidemiol 2003;157:239-48.

11 Howe GR, Zablotska LB, Fix JJ, Egel J, Buchanan J. Analysis of the mortality experience amongst US nuclear power industry workers after chronic low-dose exposure to ionizing radiation. Radiat Res 2004:162:517-26.

12 Ivanov VK. Late cancer and noncancer risks among Chernobyl emergency workers of Russia. Health Phys 2007;93:470-9.

13 McGeoghegan D, Binks K, Gillies M, Jones S, Whaley S. The noncancer mortality experience of male workers at British Nuclear Fuels plc, 1946-2005. Int J Epidemiol 2008;37:506-18.

14 Muirhead CR, O'Hagan JA, Haylock RG, Phillipson MA, Willcock T, Berridge GL, et al. Mortality and cancer incidence following occupational radiation exposure: third analysis of the national registry for radiation workers. Br / Cancer 2009;100:206-12.

15 Vrijheid M, Cardis E, Ashmore P, Auvinen A, Bae JM, Engels H, et al. Mortality from diseases other than cancer following low doses of ionizing radiation: results from the 15 -Country Study of nuclear industry workers. Int J Epidemiol 2007;36:1126-35.

16 Davis FG, Boice JD Jr, Hrubec Z, Monson RR. Cancer mortality in a radiation-exposed cohort of Massachusetts tuberculosis patients. Cancer Res 1989;49:6130-6.

17 Berrington A, Darby SC, Weiss HA, Doll R. 100 years of observation on British radiologists: mortality from cancer and other causes 1897 . 1997. Br / Radiol 2001:74:507-19.

18 Bolotnikova MG, Koshurnikova NA, Komleva NS, Budushchev EB, Okatenko PV. Mortality from cardiovascular diseases among male workers at the radiochemical plant of the "Mayak" complex. Sci Total Environ 1994;142:29-31

19 Kreuzer M, Kreisheimer M, Kandel M, Schnelzer M, Tschense A, Grosche B. Mortality from cardiovascular diseases in the German uranium miners cohort study, 1946-1998. Radiat Environ Biophys 2006;45:159-66.

20 Preston DL, Shimizu Y, Pierce DA, Suyama A, Mabuchi K. Studies of mortality of atomic bomb survivors. Report 13: solid cancer and noncancer disease mortality: 1950-1997. Radiat Res 2003;160:381-407.

21 Cullings HM, Fujita S, Funamoto S, Grant EJ, Kerr GD, Preston DL. Dose estimation for atomic bomb survivor studies: its evolution and present status. Radiat Res 2006;166:219-54.

22 Preston DL, Pierce DA, Shimizu Y, Cullings HM, Fujita S, Funamoto S, et al. Effect of recent changes in atomic bomb survivor dosimetry on cancer mortality risk estimates. Radiat Res 2004;162:377-89.

23 Breslow NE, Day N. Statistical methods in cancer research. Vol II: the design and analysis of cohort studies. Oxford University Press, 1987.

24 Preston DL, Lubin J, Pierce D. EPICURE: risk regression and data analysis software. HiroSoft International Corporation, 1991.

25 Preston DL, Ron E, Tokuoka S, Funamoto S, Nishi N, Soda M, et al. Solid cancer incidence in atomic bomb survivors: 1958-1998. Radiat Res 2007;168:1-64

26 Akaike H. A new look at statistical model identification. IEEE Trans Automat Control 1974;19:716-23.

27 Ron E, Carter R, Jablon S, Mabuchi K. Agreement between death certificate and autopsy diagnoses among atomic bomb survivors. Epidemiology 1994;5:48-56.

28 Shimizu Y, Kato H, Schull W], Hoel DG. Studies of the mortality of A bomb survivors. 9. Mortality, 1950-1985: part 3. Noncancer mortality based on the revised doses (DS86). Radiat Res 1992;130:249-66. 
29 Shimizu Y, Pierce DA, Preston DL, Mabuchi K. Studies of the mortality of atomic bomb survivors. Report 12, part II. Noncancer mortality: 1950-1990. Radiat Res 1999;152:374-89.

30 Yamada M, Wong FL, Fujiwara S, Akahoshi M, Suzuki G. Noncancer disease incidence in atomic bomb survivors, 1958-1998. Radiat Res 2004;161:622-32.

31 Robertson TL, Shimizu Y, Kato H, Kodama K, Furonaka H, Fukunaga Y, et al. Incidence of stroke and coronary heart disease in atomic bomb survivors living in Hiroshima and Nagasaki, 1958-1974. Radiation Effects Research Foundation, 1979. (RERF technical report no 12-79.)

32 Sasaki H, Wong FL, Yamada M, Kodama K. The effects of aging and radiation exposure on blood pressure levels of atomic bomb survivors. J Clin Epidemiol 2002;55:974-81.

33 Kasagi F, Kodama K, Yamada M, Sasaki H, Akahoshi M. An association between the prevalence of isolated hypertension and radiation dose in the Adult Health Study. Nagasaki Med 1992;67:479-82.

34 Wong FL, Yamada M, Sasaki H, Kodama K, Hosoda Y. Effects of radiation on the longitudinal trends of total serum cholesterol levels in the atomic bomb survivors. Radiat Res 1999;151:736-46.

35 Yamada M, Naito K, Kasagi F, Masunari N, Suzuki G. Prevalence of atherosclerosis in relation to atomic bomb radiation exposure: an RERF Adult Health Study. Int J Radiat Biol 2005;81:821-6.

36 Neriishi K, Nakashima E, Delongchamp RR. Persistent subclinical inflammation among A-bomb survivors. Int J Radiat Biol 2001;77:475-82.

37 Hayashi T, Morishita Y, Kubo Y, Kusunoki Y, Hayashi I, Kasagi F, et al. Long-term effects of radiation dose on inflammatory markers in atomic bomb survivors. Am J Med 2005;118:83-6.

38 Hayashi T, Kusunoki Y, Hakoda M, Morishita Y, Kubo Y, Maki M, et al. Radiation dose-dependent increases in inflammatory response markers in A-bomb survivors. Int J Radiat Biol 2003;79:129-36.

39 Kusunoki Y, Hayashi T. Long-lasting alterations of the immune system by ionizing radiation exposure: implications for disease development among atomic bomb survivors. Int J Radiat Biol 2008;84:1-14.

40 Kusunoki Y, Kyoizumi S, Hirai Y, Suzuki T, Nakashima E, Kodama K, et al. Flow cytometry measurements of subsets of T, B and NK cells in peripheral blood lymphocytes of atomic bomb survivors. Radiat Res 1998;150:227-36.

41 Kusunoki Y, Kyoizumi S, Yamaoka M, Kasagi F, Kodama K, Seyama T. Decreased proportion of CD4 T cells in the blood of atomic bomb survivors with myocardial infarction. Radiat Res 1999;152:539-43.

42 Pierce DA, Vaeth M, Shimizu Y. Selection bias in cancer risk estimation from A-bomb survivors. Radiat Res 2007;167:735-41.

43 Reed DM. The paradox of high risk of stroke in populations with low risk of coronary heart disease. Am J Epidemiol 1990;131:579-88.

44 Hancock SL, Tucker MA, Hoppe RT. Factors affecting late mortality from heart disease after treatment of Hodgkin's disease. JAMA 1993;270:1949-55.
45 Doody MM, Lonstein JE, Stovall M, Hacker DG, Luckyanov N, Land CE. Breast cancer mortality after diagnostic radiography: findings from the US Scoliosis Cohort Study. Spine 2000;25:2052-63.

46 Darby SC, Reeves G, Key T, Doll R, Stovall M. Mortality in a cohort of women given $\mathrm{x}$-ray therapy for metropathia haemorrhagica. Int J Cancer 1994; 56:793-801.

47 Alderson MR, Jackson SM. Long term follow-up of patients with menorrhagia treated by irradiation. Br / Radiol 1971;44:295-8.

48 Matanoski GM, Seltser R, Sartwell PE, Diamond EL, Elliott EA. The current mortality rates of radiologists and other physician specialists: specific causes of death. Am J Epidemiol 1975;101:199-210.

49 Azizova TV, Muirhead CR, Durzhinina MB, Grigoryeva ES, Vlasenko EV, Sumina MV, et al. Non-cancer effects in the cohort of workers of the first Russian nuclear facility. In: Late health effects of ionizing radiation: bridging the experimental and epidemiologic divide. Georgetown University, Washington, May 2009:46.

50 UNSCEAR. Annex B: epidemiological evaluation of cardiovascular disease and other non-cancer diseases. United Nations, 2008.

51 Little MP, Tawn EJ, Tzoulaki I, Wakeford R, Hildebrandt G, Paris F, et al. A systematic review of epidemiological associations between low and moderate doses of ionizing radiation and late cardiovascula effects, and their possible mechanisms. Radiat Res 2008;169:99-109.

52 Hendry JH, Akahoshi M, Wang LS, Lipshultz SE, Stewart FA, Trott KR. Radiation-induced cardiovascular injury. Radiat Environ Biophys 2008;47:189-93.

53 Schultz-Hector S, Trott KR. Radiation-induced cardiovascular diseases: is the epidemiologic evidence compatible with the radiobiologic data? Int J Radiat Oncol Biol Phys 2007;67:10-8.

54 Basavaraju SR, Easterly CE. Pathophysiological effects of radiation on atherosclerosis development and progression, and the incidence of cardiovascular complications. Med Phys 2002;29:2391-403.

55 Ross R. Atherosclerosis-an inflammatory disease. N Engl J Med 1999;340:115-26.

56 Stewart FA, Heeneman S, Te Poele J, Kruse J, Russell NS, Gijbels M, et al. Ionizing radiation accelerates the development of atherosclerotic lesions in ApoE-/- mice and predisposes to an inflammatory plaque phenotype prone to hemorrhage. Am J Pathol 2006;168:649-58.

57 Fajardo LF. Is the pathology of radiation injury different in small vs large blood vessels? Cardiovasc Radiat Med 1999;1:108-10.

58 Hall EJ, Brenner DJ. Cancer risks from diagnostic radiology. BrJ Radiol 2008;81:362-78.

59 Mettler FA Jr, Wiest PW, Locken JA, Kelsey CA. CT scanning: patterns of use and dose. J Radiol Prot 2000;20:353-9.

Accepted: 8 September 2009 